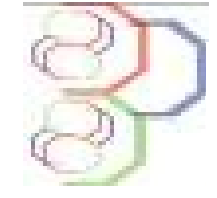

\title{
Journal of Applied Biosciences 66:5162-5172
}

ISSN 1997-5902

\section{Utilisation des Gibbérellines comme Facteur de Remodelage du cycle fructifère du citronnier 'Euréka' (Citrus limon L.)}

\author{
Malek BEN KHELIL_1, Rym BOUHLAL² et Rachid HELLALI1 \\ 'Département d'Agronomie et de Biotechnologies Végétales, Institut National Agronomique de Tunisie, 43 Avenue \\ Charles Nicole 1082, cité Mahrajène -Tunis- Tunisie ; '2Laboratoire d'Horticulture. Institut National de la Recherches \\ Agronomique de Tunisie. Rue Hédi Karray - 2049- Ariana - Tunisie.
}

Email : malekbenkhelilaf@yahoo.fr Tél : 71287110 fax : 0021671799391

Original submitted in on $25^{\text {th }}$ March 2013 Published online at www.m.elewa.org on 30th June 2013.

https://dx.doi.org/10.4314/jab.v66i0.95013

\section{RÉSUMÉ}

Objectif : Cette étude de deux années a été menée afin d'essayer de remodeler la répartition des récoltes des citronniers "Euréka" (Citrus limon L.) dans le but d'approvisionner le marché local tunisien au printemps et en été, où la demande est la plus élevée, par l'utilisation de l'acide gibbérellique $\left(\mathrm{GA}_{3}\right)$.

Méthodologie et Résultat : Deux traitements de $\mathrm{GA}_{3}$ à 20 et à 40 ppm ont été effectués en Novembre, période correspondant à la l'induction florale printanière, dans le but d'accentuer la floraison estivale et automnale, et par conséquent obtenir une production au printemps suivant et surtout en été de l'année d'après. Les traitements au $\mathrm{GA}_{3}$ effectués en automne ont permis de retarder le développement des boutons floraux printaniers de deux semaines par rapport aux arbres témoins. En outre une diminution significative de la floraison printanière a été enregistrée pour le traitement à 40 ppm (- 25\%) ce qui a influencé la production hivernale l'année suivante. Ainsi une corrélation positive a été révélée entre le taux de nouaison des fleurs estivales et automnales avec le niveau de concentration de $\mathrm{GA}_{3}$ apporté. En effet, on a pu constater une amélioration significative du taux de nouaison des fleurs estivales $(+11 \%)$ et des fleurs automnales $(+20 \%)$ des arbres qui ont reçu les traitements à 40 ppm par rapport aux arbres témoins. De plus, un remodelage de la répartition des récoltes a été réalisé à la 2 ème année de traitement, par un transfert de la récolte hivernale vers les récoltes printanières et estivales et les effets ont été plus prononcés pour le traitement à $40 \mathrm{ppm}$. Ce traitement avait augmenté significativement la production printanière de $+4.9 \%$ et estivale de $+15.9 \%$ par rapport au témoin. Egalement une réduction de $-20,8 \%$ de la production d'hiver, comparativement aux arbres témoins, a été observée.

Conclusion et application: Cette étude a permis de remodeler le cycle fructifère du citronnier Euréka par l'utilisation de l'acide gibbérellique pour mieux répondre aux besoins du marché local au printemps et en été. Mots clés : Citronnier 'Euréka', floraison, productions, remodelage, acide gibbérellique. 


\title{
Gibberellin as a factor in remodeling fruiting cycle of 'Eureka' lemon (Citrus limon L.) Trees
}

\begin{abstract}
Objective: A two years study was carried out to determine the possible effects of gibberellic acid $\left(G A_{3}\right)$ on the harvest pattern remodeling of 'Eureka' lemon tree (Citrus limon L.) produced for Tunisian (local) market supply in spring and summer .

Methodology and results: Two treatments with $\mathrm{GA}_{3}$ at 20 and 40 ppm was applied in November, at spring floral bud inductive period, in the purpose of accentuating the summer and autumn bloom and consequently obtain production in the following spring and especially in summer. $\mathrm{GA}_{3}$ treatments applied in autumn showed a delay in spring flower bud sprouting of two weeks in comparison to control trees. Also, a significant decrease in spring flowering was observed for 40 ppm treatment (-25\%) that influenced the winter cropping pattern the following year. The rate of fruit set for summer and autumn bloom showed an increasing tendency as the level concentration of $\mathrm{GA}_{3}$ had increased. In fact, a significant improvement of the rate of summer flower $(+11 \%)$ and autumn flowers $(+20 \%)$ of trees that received treatments of 40ppm compared to control trees was observed. In addition, a remodelling of the distribution of harvest pattern was conducted in the second year of treatment by a transfer of the winter harvest to the spring and summer one and the effects were most pronounced at 40-ppm treatment. This treatment had increased the spring and summer production respectively by $4.9 \%$ and $15.9 \%$. Also a reduction of $-20.8 \%$ of winter production, compared to control trees was observed.

Conclusion and Application: This study remodel the fruiting cycle of Eureka lemon by using gibberellic acid to fulfill the requirements of the local market in the spring and summer.
\end{abstract}

Key word: Lemon tree 'Eureka', flowering, production, remodelling, gibberellic acid.

\section{INTRODUCTION}

La production des Citrus a considérablement augmenté ces deux dernières décades et a atteint 100 Millions de tonnes. En Tunisie, la superficie agrumicole est d'environ 21.000 ha plantés par 6,4 millions de pieds de différentes variétés contre 16.100 ha en 1999 d'où une augmentation de $26 \%$. La production agrumicole tunisienne tient la gème position en Méditerranée avec 220.000 tonnes en moyenne/an au cours des dernières années, représentant près de $1,2 \%$ de la production méditerranéenne et dans laquelle le citronnier couvre $16 \%$ de l'effectif total des agrumes (Lebdi Grissa, 2010). Cependant, la production du citronnier est confrontée à un grand problème dû à une baisse de la production estivale qui représente $22 \%$ seulement de la production annuelle du citronnier. Cette période représente la période « de pointe » pour le citronnier où la demande du marché est la plus élevée. De plus, la technique de forçage utilisée pour remodeler la répartition des récoltes des citronniers et qui consiste à faire un arrêt des irrigations en juin-juillet des « citronniers », pour les reprendre en Août ce qui ramène les arbres à donner une floraison groupée fin Août - début Septembre, pour avoir une récolte importante en été, affecte la vigueur et la longévité des plants (Calabrese et al., 1981).

L'utilisation de l'acide gibbérellique comme régulateur de croissance s'est montrée très bénéfique pour agir sur la répartition des récoltes et retarder leur maturation sur plusieurs espèces : chez les clémentiniers (Blondel et al., 1978), les pamplemoussiers (Porat et al., 2001), les cerisiers (Westwood,1980), l'avocatier (Garcia et Lovatt ,2000) et les citronniers (Martin and Harty, 1991; Faber ,1999). Ces auteurs ont noté un échelonnement de la récolte des citronniers de la période hivernale à la période estivale et automnale suite aux traitements à l'acide gibbérellique. Ainsi dans notre étude nous allons tester l'effet des traitements au GA3 sur le remodelage des productions saisonnières du citronnier et la maturation du fruit afin d'avoir plus de flexibilité dans la récolte et la commercialisation des citrons et 
répondre ainsi aux demandes du marché local Tunisien.

MATÉRIEL ET MÉTHODES

Mise en place de l'essai : Dans cette étude des essais ont été menés dans un verger de citronniers (Citrus limon L.) de la variété 'Euréka' connu sous le nom de Citronnier des 4 saisons par sa floraison ininterrompue sur toute

l'année (Fig.1), situé à « El Gobba » au gouvernorat de Nabeul (Nord Est de la Tunisie), âgés de 6 ans et plantés avec une densité de $4 \mathrm{~m}^{*} 6 \mathrm{~m}$.

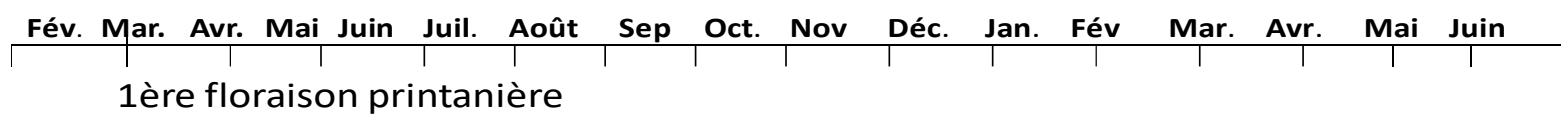

Développement des fruits de la 1ére floraison Récolte hivernale

2ème floraison estivale

Développement des fruits de la 2ème floraison Récolte printanière

3ème floraison automnale

Développement des fruits de la 3ème floraison

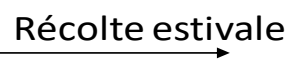

Figure1. Modèle de phénologie du citronnier « Euréka » (Ben khelil, 2005)

Le modèle expérimental adopté est un dispositif complètement aléatoire comprenant 2 niveaux de traitements (20ppm et 40ppm). On a choisi 9 arbres/ traitement au hasard, soit 27 arbres témoins et traités. Les arbres ont été choisis pour l'uniformité de leur taille, vigueur et charge en fruit. Les solutions de l'acide gibbérellique sont préparées à partir de Berlex tablets (Chaque comprimé contient 0,92 grammes (nominal) de l'acide gibbérellique (GA3). Les pulvérisations des gibbérellines ont été effectuées sur toute la frondaison de l'arbre en ajoutant du mouillant 'agral 90' (oxyde d'éthylène alkylphénolique) à raison de $0.5 \%$ par I d'eau. Nous avons traité à la dose de 0ppm :ToN1, 20ppm (2g pour 100l d'eau ) T1, 40ppm (4g pour 100l d'eau ) T2 en Novembre2003 au stade induction florale printanière, où les fruits de la récolte hivernale sont aux $1 / 2,3 / 4$ de leur calibre final. On a renouvelé les traitements sur les mêmes pieds en Novembre 2004 avec les doses Oppm :ToN2, 20ppm : T3, 40ppm :T4 aux mêmes stades précités.

Impact de la gibbérelline sur la production: La quantité de la production par pied des différentes récoltes du citronnier (hivernale, printanière et estivale) a été déterminée. Elle a été évaluée par la pesée des fruits à la récolte en totalisant pour chaque pied les quantités produites. Par la suite, on a déterminé pour chaque traitement la production totale moyenne par pied.

Impact de la gibbérelline sur la coloration : De même on a suivi la coloration des citrons avant récolte en choisissant une branche de la direction Sud-ouest portant des fruits verts et jaunes. Sur cette branche, on a déterminé le pourcentage des fruits colorés. Cette observation a été effectuée par un éventail contenant 5 graduations allant de la classe $A$ : les fruits ayant une couleur verte à $100 \%$, classe $B(75 \%$ vert ; $25 \%$ jaune), Classe C ( $50 \%$ vert ; $50 \%$ jaunes), Classe D ( $75 \%$ jaune ; $25 \%$ vert) Classe $E$ (100\%jaune).

Impact de la gibbérelline sur les paramètres de végétation et de fructification: Les paramètres de végétation et de fructification tels que: l'élongation moyenne de la pousse saisonnière mixte et végétative, la surface foliaire, le nombre de boutons floraux, le taux de nouaison (TN) ont été déterminés régulièrement en choisissant trois rameaux de la direction sud ouest de l'arbre. La surface foliaire a été mesurée à l'aide du planimètre sur un lot de 45 feuilles / traitement, prélevées au hasard des différentes pousses saisonnières (printanière, estivale et automnale). L'élongation 
moyenne de la pousse saisonnière mixte (fructifère) et végétative (non fructifère) est déterminée par la mesure en $\mathrm{cm}$ des pousses issues des bourgeons mixtes ou végétatifs. Puis on fait la somme de ces mesures qu'on divise par le nombre de pousses de chaque brindille (végétative ou fructifère) pour avoir la longueur moyenne de la pousse. Le taux de nouaison (TN) est défini par le pourcentage de nombre de fleurs nouées par rapport aux RÉSULTATS ET DISCUSSION

Effet de la gibbérelline sur la coloration et la maturation des citrons: Le retard de maturation des citrons a été constaté par le suivi de la coloration des fruits. Le pourcentage des fruits verts pour les arbres qui ont reçu les traitements T1 et T2 est resté le même tout le long de l'essai, alors que celui du témoin ToN1 a augmenté. De même, le pourcentage des citrons verts (Classe A) de la récolte hivernale 2003-2004 est resté le même pour les citronniers qui ont reçus les traitements T1 et T2 par contre ceux du témoin ToN1 ont changé de coloration au fur et à mesure qu'on s'approchait de la maturité en passant de $30 \%$ à $16 \%$ (Fig.2). Les citrons ayant une couleur vert jaunâtre appartenant à la classe $B$ fleurs $\begin{gathered}\text { totales, } \\ \mathrm{TN}(\%)=\frac{\text { nombre.de.fleurs.nouées }}{\text { nombre.total.de.fleurs }}\end{gathered} \times 100$

Analyses statistiques L'ensemble des mesures a fait l'objet d'une analyse de la variance à un seul facteur au seuil de risque de $5 \%$. L'analyse statistique a été effectuée grâce au logiciel STATICF (ver.V).

ont subi un reverdissage où on a noté une augmentation du pourcentage de $13 \%$ à $19 \%$ pour T3 et de $13 \%$ à $23 \%$ pour T4, alors que le témoin il a diminué de $10 \%$ entre les premières dates 28-01-2004 au 12-02-2004 (Fig.2). De même un reverdissage a été constaté pour les classes $D$ et $E(100 \%$ fruits jaunes) (Fig.2). Ceci rejoint les travaux de Coggins et al. (1964); Kuraoka et al.(1977) ; Blondel et al. (1978) ; El-Zeftawi (1980) et Faber (1999) qui ont démontré que les gibbérellines peuvent engendrer une réduction de la vitesse de dégradation de la chlorophylle et le retard du développement des pigments caroténoïdes et par la suite de ralentir le processus de vieillissement des tissus de la peau.

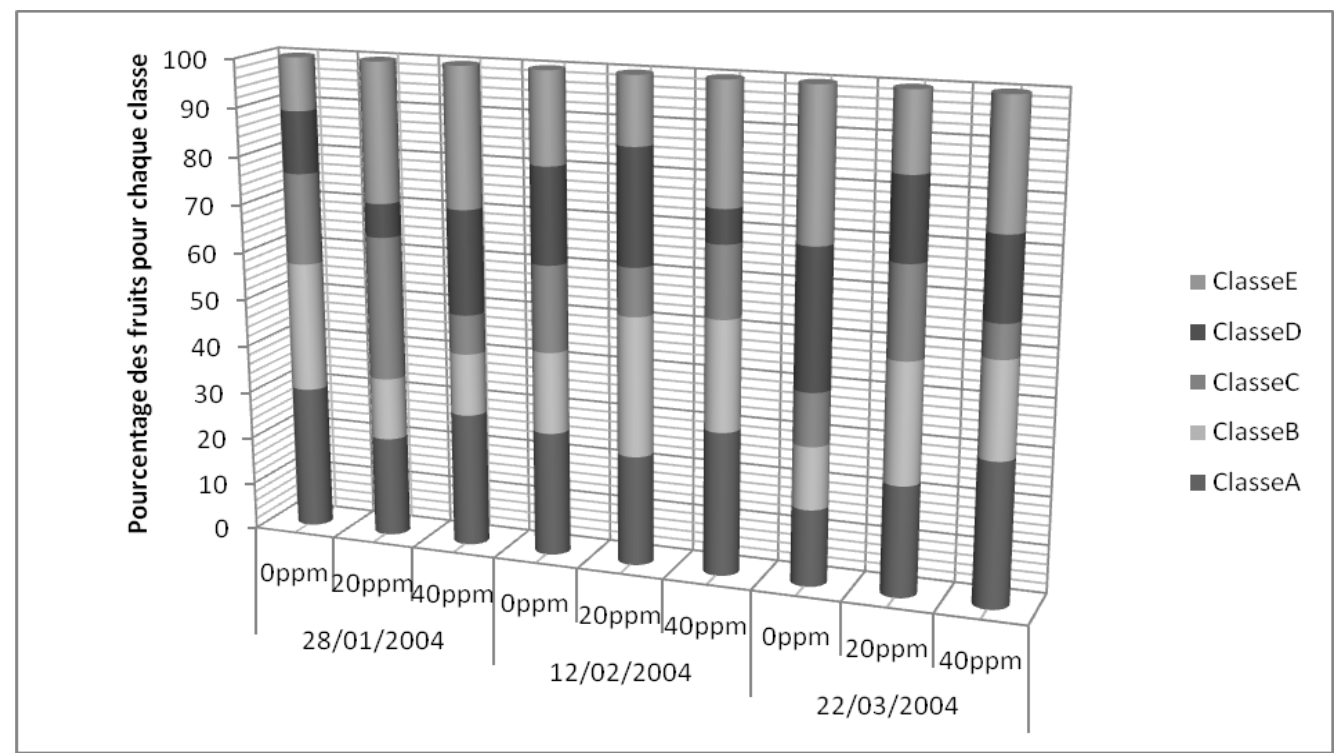

Classe A : $100 \%$ fruit vert, Classe B :75\%vert, $25 \%$ jaune, Classe C :50\%vert,50\% jaune ;Classe D : $75 \%$ jaune, $25 \%$ vert, Classe E :100\%jaune

Fig. 2 : Effet des traitements d'acide gibbérellique (ToN1=0ppm, T1=20ppm, T2=40ppm ) à la 1 ère année de traitement sur la coloration des citrons « Euréka »

Effet de la gibbérelline sur les paramètres de
végétation et de fructification Effet de la gibbérelline sur la surface foliaire: Les pulvérisations effectuées en Novembre 2003 semblent avoir un effet sur la surface foliaire moyenne (qui reflète l'activité photosynthétique) des différentes pousses saisonnières des citronniers 'Euréka' (Fig.3). En effet, cette surface a diminué significativement pour la pousse printanière 2004 qui était de $24.3 \mathrm{~cm}^{2}$ pour traitement T2 par rapport au témoin ToN1 $\left(37.4 \mathrm{~cm}^{2}\right) \quad$ (Fig.3). 
Cependant, une légère augmentation est constatée pour la pousse estivale et automnale des arbres traités par rapport aux arbres témoins. La diminution de la surface foliaire observée sur les feuilles de la pousse printanière est attribuée aux effets immédiats des apports exogènes des gibbérellines. Par ailleurs, Sidahmed (1986) a montré que les pulvérisations de GA3 sur le feuillage du 'lime' ont induit une légère diminution de la surface foliaire et une augmentation de la longueur des tiges.

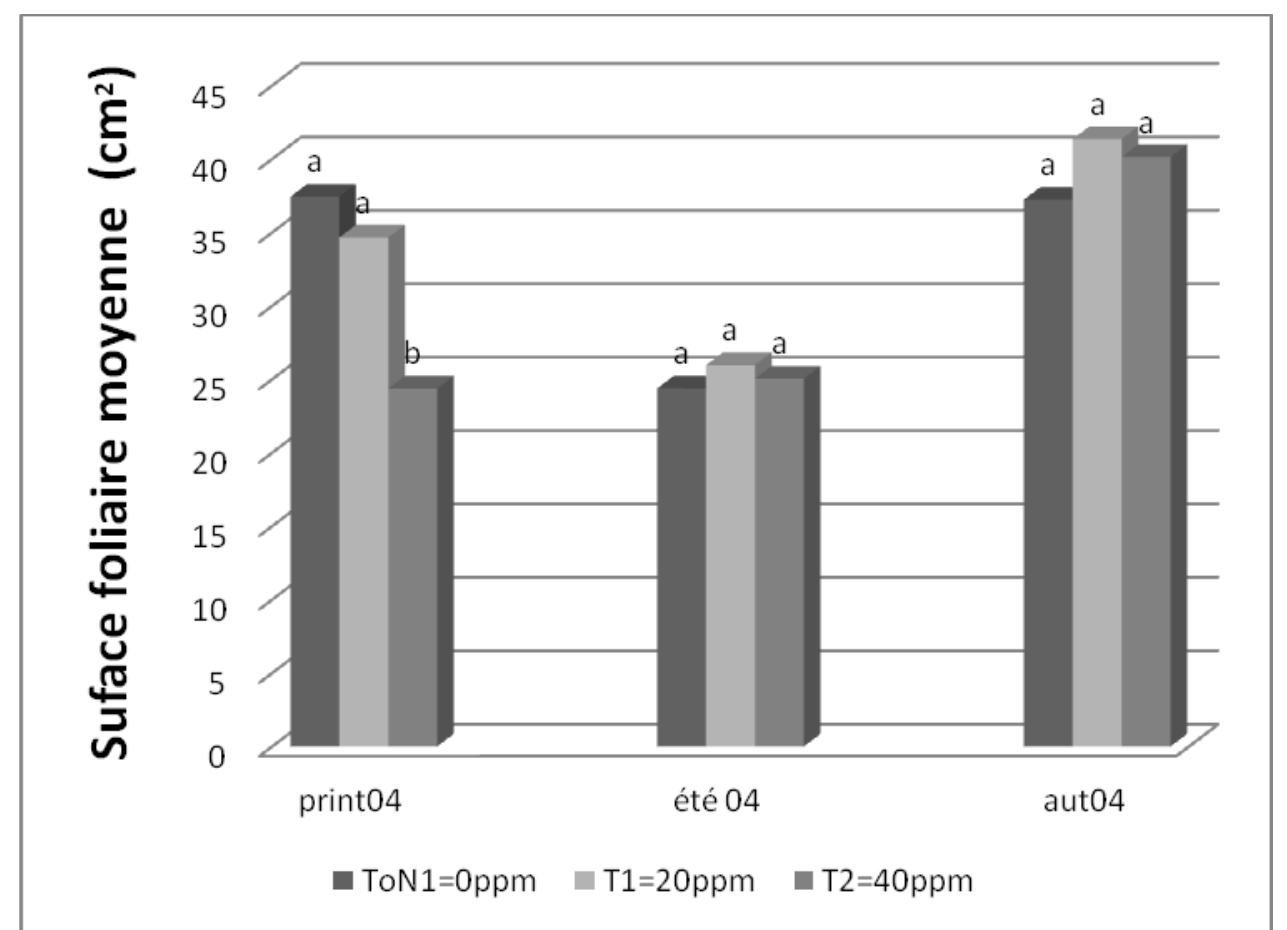

Fig.3 : Surface foliaire moyenne des différentes pousses saisonnières des citronniers traités au GA3

Effet de la gibbérelline sur l'élongation moyenne des pousses végétatives saisonnières: Les traitements au GA3 effectués en Novembre au moment de l'induction florale printanière semblent agir sur l'élongation moyenne de la pousse végétative de celle-ci. En effet, l'analyse statistique a montré que l'élongation moyenne de la pousse végétative printanière a significativement augmenté pour les deux traitements T1 et T2 par rapport à celle du témoin (Fig.4.).

Concernant l'élongation végétative de la pousse estivale, on remarque qu'elle a augmenté légèrement pour les arbres traités par rapport au témoin. Cette augmentation de l'élongation est non significative par rapport au témoin au seuil de 5\%. En effet l'élongation moyenne était de 2.2 $\mathrm{cm}$ pour le témoin alors qu'elle était légèrement supérieure pour le traitement T2 $(2.6 \mathrm{~cm})$. L'élongation moyenne végétative automnale a été la plus élevée pour le traitement à 40ppm par rapport à celle du témoin et égale à $7.8 \mathrm{~cm}$. L'analyse statistique de la variance ne montre pas de différence significative par rapport à celle du témoin. 


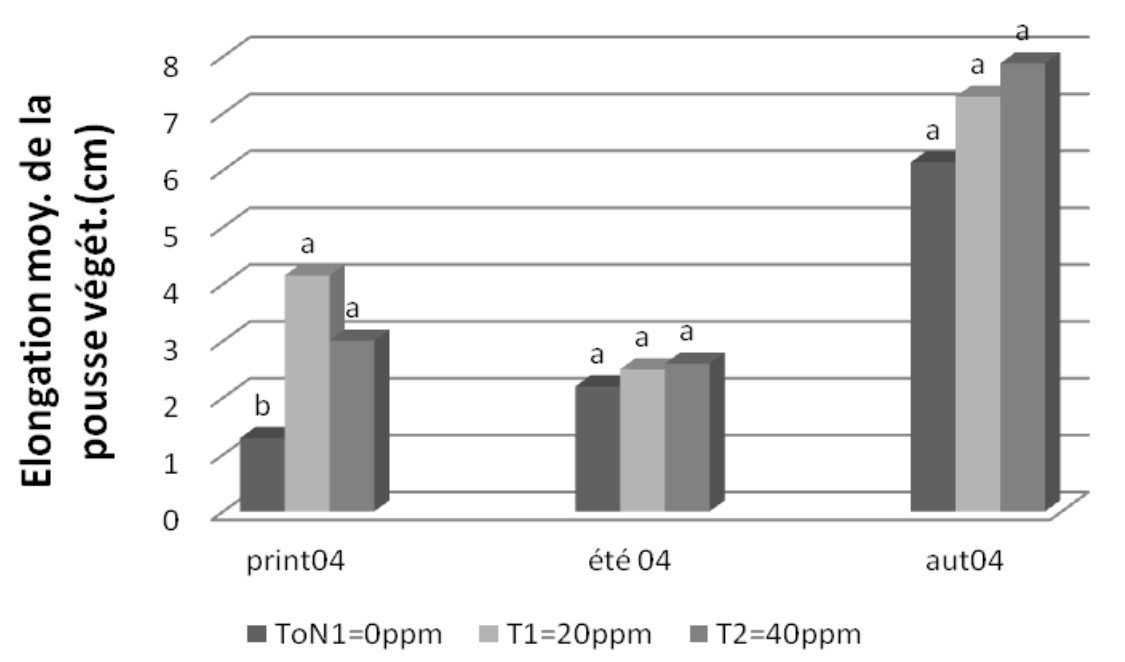

Fig. 4 : Élongation moyenne des pousses végétatives saisonnières des citronniers traités au GA3

Les traitements de GA3 effectués, en Novembre, semblent agir sur l'élongation moyenne des pousses végétatives printanières qui se sont développées au dépend des pousses mixtes et ceci réside de l'effet secondaire inhibiteur des gibbérellines sur la fructification. Ces résultats concordent avec ceux de Guardiola et al. (1982) où l'application de GA3 chez les agrumes en Novembre a induit une inhibition supplémentaire résultant d'une réduction des pousses fructifères avec une augmentation des pousses végétatives, ce qui suggère la réversion de fleurs à l'apex végétatif.

Effet de la gibbérelline sur l'élongation moyenne des pousses mixtes saisonnières : L'élongation moyenne de la pousse mixte printanière, a diminué légèrement suite aux traitements de GA3 effectués en Novembre. L'analyse de la variance montre que cette diminution est non significative au seuil de 5\% (Fig.5). Les traitements aux gibbérellines semblent exercer un effet positif sur l'élongation moyenne mixte des pousses estivales et automnales. Cet accroissement était significatif pour la pousse automnale qui était égale à $3.14 \mathrm{~cm}$ pour le traitement T2 (40ppm) par rapport au témoin $(1.9 \mathrm{~cm})$. Cette amélioration de la pousse mixte a constitué une source de carbohydrates pour l'évolution du bouton floral en fruit ce qui a engendré une augmentation des productions saisonnières au printemps et à l'été l'année suivante (Tableaux 1-2).

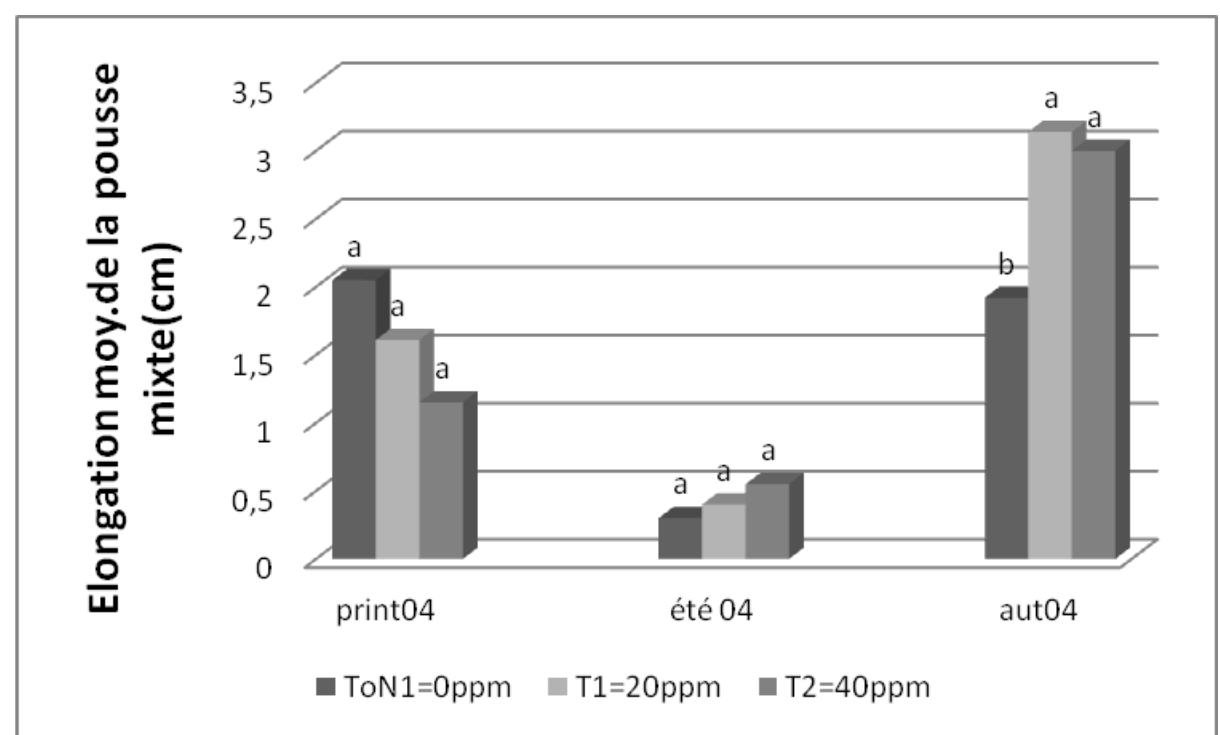

Fig. 5 : Élongation moyenne des pousses mixtes saisonnières des citronniers 'Euréka' traités au GA3 en Novembre 
Effet de la gibbérelline sur la floraison : Un retard de la floraison printanière de deux semaines a été constaté des arbres traités par les gibbérellines par rapport aux arbres témoins qui va s'échelonner et donner des fruits à la fin du printemps et au début d'été, sachant que la production finale dépend du taux de nouaison et du développement postérieur du fruit. De telles observations ont été notées sur 'Valencia' et 'Pineapple' oranges par Fidelibus et Davies (2002) qui révèlent un retard de la floraison pour les deux variétés d'agrume précitées. Ces résultats concordent aussi avec ceux hamada (1995) qui se sont orientés vers la stimulation du retardement de la maturation permettant par conséquent une plus longue conservation du fruit sur l'arbre. En effet les applications des gibbérellines au moment de l'induction florale (Novembre) ont induit une diminution du nombre de boutons floraux de la floraison printanière. Ce nombre était plus élevé pour le témoin ToN1 qui était de 6.3 alors que pour le traitement T2 (40ppm), il était de 4.8. Le taux de diminution est égal à $-25 \%$ par rapport au témoin ToN1 (Fig.6). Ces résultats concordent avec ceux de Gonzalez-Rossia et al. (2007) qui montrent que des concentrations de 0.5 ou $1.0 \mathrm{~g}$ d'acide gibbérellique par arbre ont réduit la floraison d'environ $50 \%$ dans les pêches et les nectarines respectivement. Des études récentes sur les agrumes, ont noté que l'acide gibbérellique (GA3) appliqué à la période d'induction florale réduit de manière significative l'intensité de la floraison. Cet effet est utilisé pour améliorer la nouaison des cultivars parthénocarpiques qui ont tendance à fleurir à profusion. Ces résultats suggèrent que les gibbérellines inhibent la floraison chez les agrumes en réprimant l'expression CIFT dans les feuilles (Munoz- Fambuena et al., 2012) .

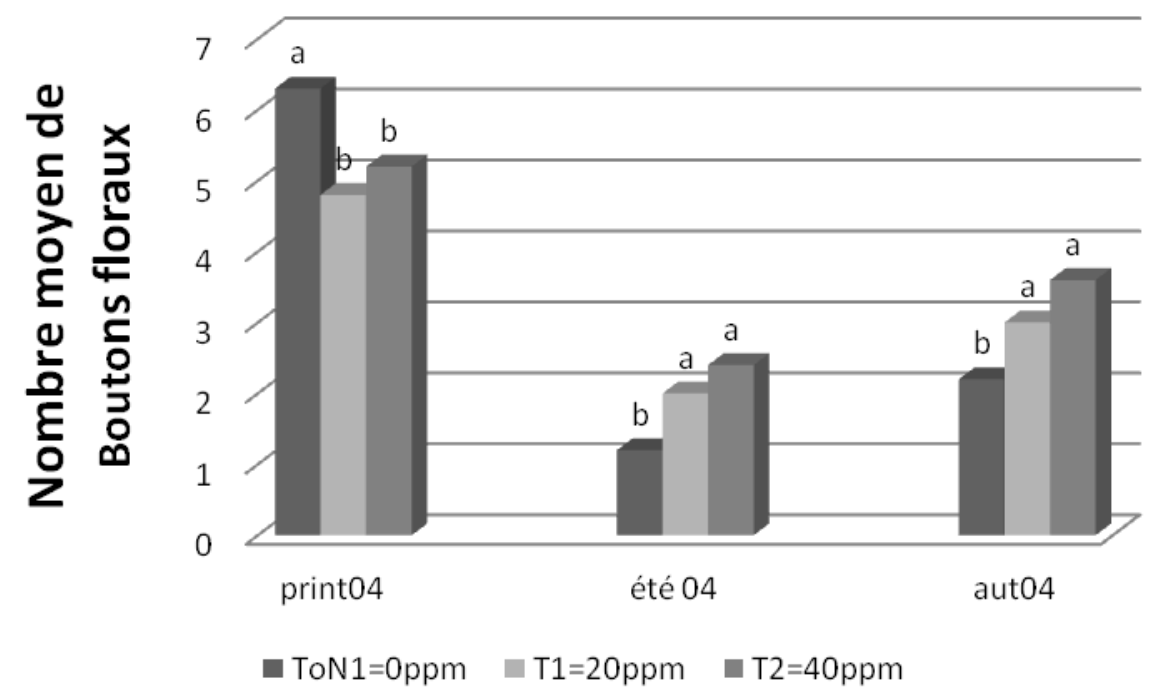

Fig.6 : Nombre moyen de boutons floraux des citronniers « Euréka » traités au $\mathrm{GA}_{3}$ en Novembre

Effet de la gibbérelline sur le taux de Nouaison : Le taux de nouaison des fleurs issues de la floraison printanière, s'est avéré moins élevé pour le traitement T1 (20ppm) qui était de $6.1 \%$ et de $8.2 \%$ pour le traitement T2 (40ppm) par rapport au témoin ToN1 (12.8\%) (Fig.7). Le taux de nouaison des fleurs issues de la floraison estivale, a augmenté significativement pour les deux traitements $\mathrm{T} 1$ et $\mathrm{T} 2$ respectivement qui étaient égaux à $61 \%$ et $63 \%$ par rapport au témoin (47\%) (Fig.7). Par contre ce pourcentage était légèrement supérieur au témoin pour le traitement T1 (56\%) pour les fleurs issues de la floraison automnale et a augmenté significativement pour le traitement T2 qui était égal à 72\% (Fig.7). Ainsi, on a pu constater une amélioration plus significative du taux de nouaison des fleurs estivales $(+11 \%)$ et des fleurs automnales $(+20 \%)$ des arbres qui ont reçu les traitements à $40 \mathrm{ppm}$ par rapport aux arbres témoins. 


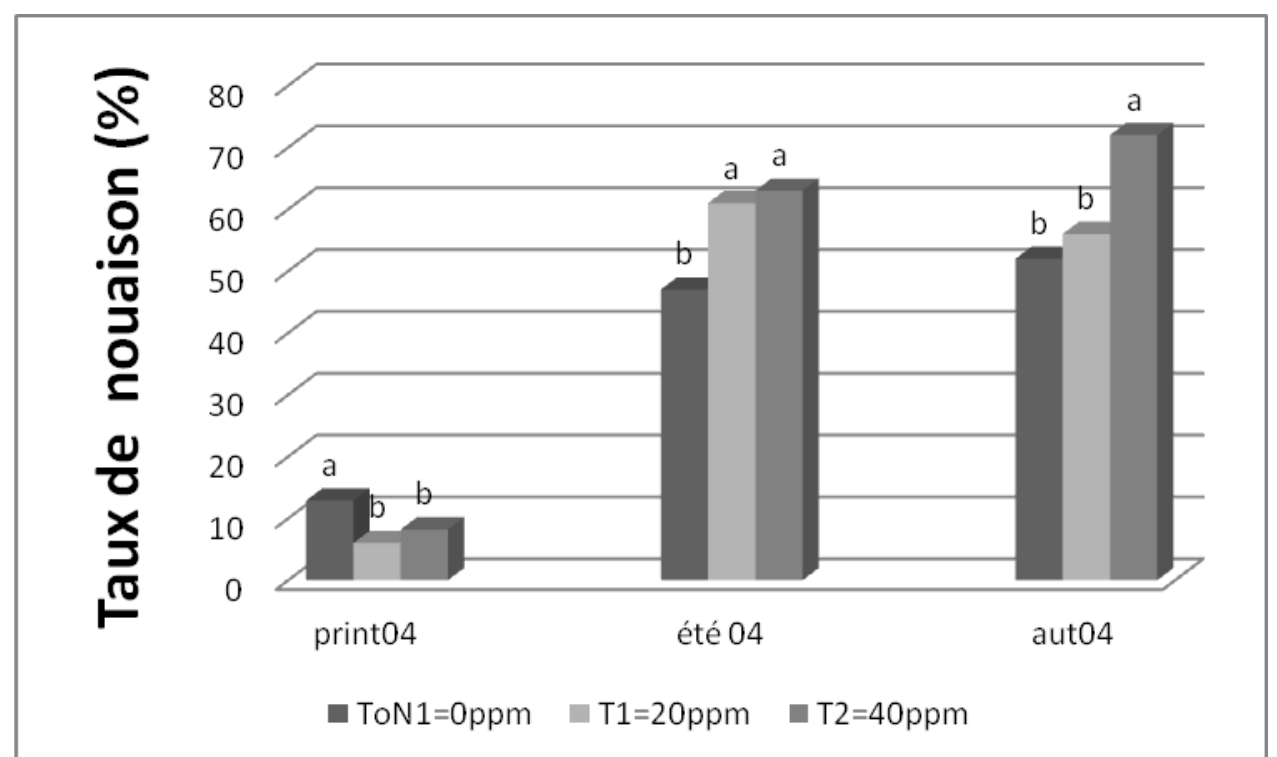

Fig. 7 : Taux de nouaison saisonniers des citronniers « Euréka » traités au $\mathrm{GA}_{3}$ en Novembre

Effet de la gibbérelline sur l'échelonnement des productions saisonnières: Un remodelage de la répartition des récoltes a été réalisé à la 2ème année de traitement aux gibbérellines, par un transfert de la récolte hivernale vers les récoltes printanières et estivales. Cet effet semble être bénéfique pour des pulvérisations prévues pour deux années et les effets ont été plus prononcés pour le traitement à $40 \mathrm{ppm}$. En effet les traitements consécutives, effectués en Novembre pour les deux années de suivie ont permis une amélioration de la production printanière (Mi Mars 2005-début Mai 2005) observé à la 2ème année de traitement. Celle ci a augmenté de $+2.5 \mathrm{~kg} /$ pied $(+2.9 \%)$ et de $+4.4 \mathrm{~kg} /$ pied $(+4.9 \%)$ respectivement pour les traitements $\mathrm{T} 3$ et $\mathrm{T} 4$ significativement par rapport au témoin ToN2 au seuil de $5 \%$ (Tableaux 1-2). Egalement un accroissement significatif de la production estivale 2005 (début juillet- fin Août) pour le traitement T3 de $+11.9 \mathrm{~kg} /$ pied $(+14.1 \%)$ et $\mathrm{de}+13.6 \mathrm{~kg} /$ pied $(+15.9 \%)$ par rapport au témoin ToN2 (Tableaux 1 et 2). On a aussi noté une diminution de la production hivernale 2005 (début Décembre 2004-mi Janvier2005) pour les traitements T3 de $-14.6 \mathrm{~kg} / \mathrm{pied} \mathrm{(-}$
$17 \%$ ) et de $-17.5 \mathrm{~kg} /$ pied (-20.8 \%) pour le traitement T4 par rapport au témoin ToN2. Cette diminution de la production pour les deux traitements T3 et T4 est significative au seuil de $5 \%$ (Tableaux 1 et 2). Ces résultats semblent concorder avec ceux de Faber (1999) sur citronnier à quatre saisons où un échelonnement $a$ été réalisé de la période hivernale à la période d'été et d'automne. En effet, Le moment choisi pour l'application des gibbérellines en Novembre à la période d'induction florale printanière a eu un effet inhibiteur de celle ci, ce qui a permis une réduction de la récolte hivernale de l'année suivante et cet effet est plus marqué que la concentration d'acide gibbérellique est élevée. Plusieurs chercheurs affirment que l'application des gibbérellines quelques semaines avant la période d'induction florale (novembre-décembre) peut diminuer l'intensité de cette dernière et entraîner une diminution de la récolte suivante. Sur abricotier, Hellali et Laajimi (1998) tirent une application pratique du GA3 pour réduire l'induction florale et provoquer un éclaircissage anticipé du fruit en « return blum » afin de régulariser la production d'une année à l'autre. 
Tableau 1 : Les quantités des récoltes des citrons en fonction des différents traitements au GA3 pour chaque saison

$\begin{array}{ccc}\begin{array}{c}\text { Périodes de } \\ \text { traitements }\end{array} & \text { Concentrations } & \text { Quantités récoltées }(\mathrm{Kg} / \mathrm{pied})\end{array}$

\begin{tabular}{|c|c|c|c|c|c|}
\hline \multirow[t]{5}{*}{ Novembre 2003} & & $\begin{array}{c}\text { Récolte } \\
\text { hivernale } \\
(2003-2004)\end{array}$ & $\begin{array}{c}\text { Récolte } \\
\text { printanière } \\
(2004)\end{array}$ & $\begin{array}{l}\text { Récolte } \\
\text { estivale } \\
(2004)\end{array}$ & Total \\
\hline & \multirow[t]{2}{*}{0 ppm :ToN1 } & & & & \\
\hline & & $39.5 \mathrm{c}$ & $18.6 c^{\prime}$ & $16.1 c^{\prime \prime}$ & 74.2 \\
\hline & $20 \mathrm{ppm}: \mathrm{T} 1$ & $41.9 b$ & $19.8 c^{\prime}$ & $13.5 d^{\prime \prime}$ & 75.2 \\
\hline & $40 \mathrm{ppm}: \mathrm{T} 2$ & $43.3 a$ & $20.2 b^{\prime}$ & $12.5 \mathrm{~d} "$ & 76.0 \\
\hline \multirow[t]{4}{*}{ Novembre 2004} & & $\begin{array}{c}\text { Récolte } \\
\text { hivernale } \\
(2004-2005)\end{array}$ & $\begin{array}{l}\text { Récolte } \\
\text { printanière } \\
(2005)\end{array}$ & $\begin{array}{c}\text { Récolte } \\
\text { estivale } \\
(2005)\end{array}$ & Total \\
\hline & 0 ppm :ToN2 & 445 & $178 r^{\prime}$ & 220 "2" & \\
\hline & 20 ppm :T3 & $29.9 d$ & $20.3 b^{\prime}$ & $35.1 a^{\prime \prime}$ & 85.3 \\
\hline & $40 \mathrm{ppm}: \mathrm{T} 4$ & $27.0 \mathrm{e}$ & $22.2 a^{\prime}$ & $36.8 a "$ & 86.0 \\
\hline
\end{tabular}

${ }^{\star \star \star}$ les moyennes qui ne sont pas suivies de la même lettre sont significativement différentes au seuil de risque de $5 \%$ (test de Newman-keuls

Tableau 2 : les récoltes saisonnières des citrons en pourcentage du total des trois récoltes, et en fonction des différents traitements au GA3

\begin{tabular}{|c|c|c|c|c|c|}
\hline \multirow{2}{*}{$\begin{array}{c}\begin{array}{l}\text { Périodes de } \\
\text { traitements }\end{array} \\
\text { Novembre } \\
2003\end{array}$} & \multirow{2}{*}{ Concentrations } & \multicolumn{3}{|c|}{$\begin{array}{l}\text { Pourcentages des récoltes }(\%) \\
\end{array}$} & \multirow[b]{2}{*}{ Total } \\
\hline & & $\begin{array}{c}\text { Récolte hivernale } \\
(2003-2004)\end{array}$ & $\begin{array}{c}\text { Récolte } \\
\text { printanière } \\
(2004)\end{array}$ & $\begin{array}{c}\text { Récolte } \\
\text { estivale } \\
(2004)\end{array}$ & \\
\hline & 0 ppm : ToN1 & 540 & 250 & 210 & 100 \\
\hline & $20 \mathrm{ppm}: \mathrm{T1}$ & 55.8 & 26.3 & 17.9 & 100 \\
\hline & $40 \mathrm{ppm}: \mathrm{T} 2$ & 56.8 & 26.7 & 16.5 & 100 \\
\hline \multirow[t]{4}{*}{$\begin{array}{l}\text { Novembre } \\
2004\end{array}$} & \multirow{2}{*}{0 ppm :ToN2 } & $\begin{array}{l}\text { Récolte hivernale } \\
(2004-2005)\end{array}$ & $\begin{array}{c}\text { Récolte } \\
\text { printanière } \\
(2005)\end{array}$ & $\begin{array}{c}\text { Récolte } \\
\text { estivale } \\
(2005)\end{array}$ & Total \\
\hline & & 52.0 & 20.9 & 27.1 & 100 \\
\hline & $20 \mathrm{ppm}: \mathrm{T3}$ & 35.0 & 23.8 & 41.2 & 100 \\
\hline & $40 \mathrm{ppm}: \mathrm{T4}$ & 31.2 & 25.8 & 43.0 & 100 \\
\hline
\end{tabular}

\section{CONCLUSION}

A la lumière des résultats obtenus, suite aux pulvérisations au GA3 des doses 20 et 40 ppm effectuées en Novembre afin d'essayer de remodeler la répartition des récoltes des citrons surtout pour un approvisionnement du marché au printemps et en été, nous avons pu tirer les conclusions suivantes :

Les traitements effectués au stade induction florale printanière, où les fruits de la récolte hivernale sont aux $1 / 2,3 / 4$ de leur calibre final et en présence des fruits noués de la floraison automnale ont eu un effet de transférer une partie de la récolte hivernale 2004-2005 vers celle printanière 2005 et estivale 2005. Le remodelage de la répartition des récoltes était plus élevé et significatif pour le traitement T4 à 40ppm de GA3, où on a noté une amélioration significative de la récolte printanière2005 de $+4.9 \%$ et estivale 2005 de $+15.9 \%$ par rapport au témoin ToN2. De plus un échelonnement de la floraison a été déterminé suite aux traitements aux gibbérellines. En 
effet, un retard de la floraison printanière de deux semaines a été constaté des arbres traités par les gibbérellines par rapport aux arbres témoins qui vont s'échelonner et donner des fruits fins printemps et début d'été. Aussi, a pu constater une amélioration significative du taux de nouaison plus marqué pour le traitement à 40 ppm des fleurs estivales (+11\%) et des fleurs automnales $(+20 \%)$. Les paramètres de végétation et de fructification ont été aussi stimulés par l'apport exogène des gibbérellines effectué. Nous avons aussi constaté une compétition entre le développement de la surface foliaire

\section{RÉFÉRENCES BIBLIOGRAPHIQUES}

Ben Khelil M, 2005. Recherche de possibilité de remodelage de la répartition des différentes productions du citronnier à quatre saisons par l'utilisation de l'acide gibbérellique. Mastère. Lab. d'arboriculture fruitière. Institut National Agronomique de Tunisie (INAT). Tunis .Tunisie. $138 \mathrm{p}$.

Blondel L, Marchioni JB, Vittori F, 1978. Effets de quelques substances de croissance sur le clémentinier. Fruits. 33(12) : 853-855.

Calabrese F. and Di Marco L, 1981. Researches on the "Forzatura" of Lemon Trees. Proc. Int.Soc. Citriculture Vol.2: 520-521.

Coggins Jr CW. and Hield HZ, 1968. Plant growth regulators. In. The Citrus Industry W. Reuther $L$ D, Bachelor and HJ Webber. Vol2. Univ. Of California Berkley. P371-389

El-Zeftawi BM, 1980 .Effects of gibberellic acid and cycocel on colouring and sizing of lemon. Scientia Horticulturae .Volume 12, Issue 2, March 1980, Pages 177-181

Faber BA, 1999. Potential problems with gibberillic acid application

to

Citrus.www.research.com/sub/sub1099/fab.html. 6k. A cooperative. Publication of California. Subtropical fruit news. Vol 7.N¹.1999.

Fidelibus MW. and Davies FS, 2002. Gibberellic Acid application Timing Affects Fruit Quality of Processing Oranges. Hortscience 37(2): p 353357.

Garcia SS. and Lovatt CJ, 2000. Use of GA3 to Manipulate Flowering and Yield of Hass Avocado. J. Amer. Soc. Hort.Sci.125 (1) 25-30.

Gonzalez-Rossia D, Reig C, Juan M, Agusti M, 2007. Horticultural factors regulating effectiveness of GA3 inhibiting flowering in peaches and et de l'élongation moyenne végétative de la pousse printanière au dépend des pousses mixtes et ceci réside de l'effet secondaire inhibiteur des gibbérellines sur la fructification printanière. Cependant, une amélioration de la pousse mixte des pousses estivales et automnales a été constaté ce qui a constitué une source de carbohydrates pour l'évolution du bouton floral en fruit et a été une conséquence de l'augmentation des productions saisonnières au printemps et à l'été l'année suivante.

nectarines (Prunus persica L. Batsch). Scientia Horticulturae 111. 352-357

Guardiola JL, Monerri C, Agusti M, 1982. The inhibitory effect of gibberellic acid on flowering in citrus. Physiol plant 55:136-142.

Hamada M, 1995. Possibilité de retarder la maturation des oranges Maltaise (Citrus Sinensis Osbeck) C.V.demi sanguine par pulvérisation foliaire de GA3 et d'urée permettant l'échelonnement de leur récolte. Mémoire de 3ème cycle de spécialisation. Institut National Agronomique de Tunisie (INAT). Tunis .Tunisie.165p.

Hellali R. and Laajimi N, 1998. Eclaircissage des abricots. Fiche d'activité de recherche, dans Rapport d'activité de recherche .Tunis.Tunisie, (1998) .95p

Kuraoka TK, Iwosaki T Ishii,1977. Effects of GA3 on puffing and levels of GA3 like substances and $A B A$ in the peel of Satsuma mandarin (Citrus unshu Marc) J. Amer. Soc. Hort. Sci. 102 (1977) 651-654.

Lebdi Grissa K, 2010. Etude de base sur les cultures d'agrumes et de tomates en Tunisie. Regional Integrated Pest Management Program in the Near East GTFS/REM/070/ITA. 92p.

Martin P. and Harty A, Producing Summer.1991. Lemons. Kerikeri Horticultural Research Station, Citrus Research Seminar. In Growing lemons in Australia- a production manual 'Readers' Note. 20-22.

Munoz- Fambuena, N Mesejo, Carmen GonzálezMas CM, Iglesias DJ, Primo-Millo E, Agustí M, 2012. Gibberellic Acid Reduces Flowering Intensity in Sweet Orange [Citrus sinensis (L.) Osbeck] by Repressing CiFT Gene Expression. Journal of Plant Growth Regulation. Volume 31, Issue 4, pp 529-536. 
Porat R, Feng X, huberman M, Galili D, Goren R, Goldsmith E, 2001 . Gibberellic Acid slows Postharvest Degreening OD 'Oroblanco' citrus fruits. Hort.Sci.36 (5):937-940.

Sidahmed A, 1986.Effects of different $\mathrm{N}$ levels and gibberellic acid on growth and composition of field grown young lives. Program and abstract. Annual. Meeting of the Amer. Hort.Sci.21 (3):808.

Westwood MN, 1980. Temperate Zone Pomology .Physiology and culture $.3^{\text {rd }}$ ed. Timber press, Potland, oregan. 428p. 